\title{
MMP-14-targeted Nanoprobe for in vivo \\ Fluorescence Imaging of Rupture-prone Carotid Plaques
}

\section{Mengtao Han}

Shandong University Qilu Hospital

\section{Kaining Liu}

Shandong University Qilu Hospital

Hongqiu Xiao

Shandong University Qilu Hospital

Tao Sun

Shandong University Qilu Hospital

\section{Fei Wang}

Shandong University Qilu Hospital

\section{Yiming He}

Shandong University Qilu Hospital

Donghai Wang ( $\nabla$ drwangdonghai@sdu.edu.cn )

Shandong University Qilu Hospital https://orcid.org/0000-0001-5048-5040

\section{Research Article}

Keywords: Atherosclerosis, Macrophages, Matrix metalloproteinases-14 (MMP-14), Membrane type 1 metalloprotease (MT1-MMP), Fluorescence imaging, Nanoprobe

Posted Date: May 24th, 2021

DOI: https://doi.org/10.21203/rs.3.rs-507897/v1

License: (c) (1) This work is licensed under a Creative Commons Attribution 4.0 International License. Read Full License 


\section{Abstract}

Background: The identification of rupture-prone carotid plaques for preventing stroke remains a clinical challenge. Macrophage matrix metalloproteinase (MMP)-14, which contributes to plaque progression and destabilisation, could be a promising biomarker for plaque imaging. This study aimed to design and synthesise an MMP-14-targeted nanoprobe to noninvasively visualise the behaviour of M1 macrophages in atherosclerotic plaques.

Methods: A fluorescence molecular imaging probe (AuNPs@PEG-Peptide-Cy5.5) was constructed by covalently attaching the fluorescent dye cyanine (Cy) 5.5, an MMP-14 substrate, and polyethylene glycol (PEG) 5000-wrapped gold nanoparticles (AuNPs), and then administered via tail vein injection to carotid atherosclerosis models for in vivo fluorescence imaging. Additionally, carotid tissues and cultured macrophages were analysed for nanoprobe binding, and MMP-14 and inflammation-related marker expression was evaluated by polymerase chain reaction, western blotting, and immunohistochemistry.

Results: MMP-14 expression significantly increased with plaque progression, along with the upregulation of MMP-2 and inflammatory M1 markers, CD68 and F4/80, and significant downregulation of the M2 marker CD206. All of cell, tissue and in vivo fluorescence imaging exhibited a favourable targeting efficacy of AuNPs@PEG-Peptide-Cy5.5 for MMP-14.

Conclusions: MMP-14, a cell membrane-anchoring enzyme, can serve as a biomarker of vulnerable plaques, and MMP-14 substrate-based AuNPs@PEG-Peptide-Cy5.5, with an intense fluorescence signal after activation and good biocompatibility, can be applied to screen for and monitor plaque progression in vivo.

\section{Background}

Carotid atherosclerosis is a strong risk factor for ischemic stroke [1]. As a multifactorial and complex systemic disease, carotid atherosclerosis is characterised by the formation of atherosclerotic plaques, which is a lengthy process that involves the deposition of cholesterol, inflammation, and cell infiltration into vessel walls [2, 3]. After a plaque buildup, its enlargement may result in a severe occlusion of the lumen and cause distal hypoperfusion or rupture to embolise a distal branch, leading to morbidity and mortality [4]. Therefore, there is an urgent need to identify plaques that are prone to enlargement or rupture.

Matrix metalloproteinase (MMP)-14, a cell membrane-anchoring enzyme, has been reported to be overexpressed in vulnerable human atherosclerotic plaques [5-8]. MMP-14 can activate pro-MMP-2 [9] and pro-MMP-13 [10], as well as interact with the hyaluronate receptor CD44 to activate MMP-9 [11] to break down the extracellular matrix within the lesion, converting chronic stable plaques into acute unstable plaques, with the potential for thromboembolism [12]. Macrophages contribute to a broad range of chronic inflammatory processes and furnish most MMPs in atheromata [6,13], and therefore, many atherosclerosis-related studies have focused on macrophages. Immunohistochemical results have 
confirmed that MMP-14 is mainly codistributed with macrophages in the fragile region of rupture-prone plaques [7, 14], although other types of cells, such as smooth muscle cells, also express small amounts of MMP-14. Furthermore, MMP-14-directed macrophage invasion increases the likelihood of the rupture at the shoulder of atherosclerotic plaques $[5,15]$. Thus, MMP-14 from macrophages could be a promising biomarker for plaque imaging.

Current imaging technologies, such as computer tomography, magnetic resonance imaging, and positron emission tomography (PET), enable the determination of the severity of luminal narrowing and the assessment of plaque vulnerability at the morphological level; however, these technologies have a poor accuracy and specificity and cannot provide information about molecular signatures of rupture-prone plaques at the cellular level $[4,16]$. Consequently, more advanced imaging modes are needed to demonstrate the extent of the disease spread. In vivo molecular imaging strategies provide a way beyond the anatomy to visualise atherosclerotic inflammation, angiogenesis, apoptosis, oxidative stress, and calcification, thus overcoming the limitations of traditional imaging methods [17]. However, primary intravascular molecular imaging uses radioisotope-derivatised monoclonal antibodies, whose large sizes do not allow fast on/off rates and produce high target-to-background ratios in vivo $[18,19]$. Near-infrared (NIR) fluorescence imaging with a targeted nanoprobe is a recent and promising imaging technology that allows noninvasive visualisation of the metabolic state of various types of cells, local inflammatory responses, and intraplaque angiogenesis [20-22]. Moreover, its real-time modality, high sensitivity, and high specificity, as well as the absence of ionising radiation, make NIR fluorescence a preferred option for the diagnosis of rupture-prone plaques to assist in clinical decision making [20,23].

The main aim of this study was to investigate and validate the mechanism by which MMP-14, a promising biomarker of $\mathrm{M} 1$ macrophages, contributes to the progression and rupture of atherosclerotic plaques. For this purpose, an MMP-14-targeted nanoprobe, AuNPs@PEG-Peptide-Cy5.5, was designed and synthesised to noninvasively visualise the behaviour of M1 macrophages in atherosclerotic plaques.

\section{Methods}

\subsection{Synthesis of AuNPs@PEG-Peptide-Cy5.5}

A cyanide dye (Cy) 5.5-labelled Gly-Arg-lle-Gly-Phe-Leu-Arg-Thr-Ala-Lys-Gly-Gly peptide (5 mg, $0.0028 \mathrm{mmol}$ ) was synthesised as previously reported[24, 25], and commercially available SHpolyethylene glycol (PEG)-COOH-modified Au nanoparticles (NPs) were purchased from XFNANO (Nanjing, Jiangsu, China). Next, $10 \mu \mathrm{L}$ of $0.1 \%$ sodium dodecyl sulphate (SDS) and $0.5 \mathrm{~mL}$ of the Cy5.5labelled peptide $(2.453 \mathrm{mg} / \mathrm{mL})$ were added to $10 \mathrm{~mL}$ of the AuNPs $[0.1 \mathrm{mg} / \mathrm{mL}$, dispersed in $2-(N-$ morpholino) ethanesulphonic acid], and stirred for $30 \mathrm{~min}$. Thereafter, $0.5 \mathrm{mg}$ of 1 -ethyl-3-(3dimethylaminopropyl) carbodiimide $(10 \mathrm{mg} / \mathrm{mL})$ was added, and the mixture was stirred at room temperature overnight. At the end of the reaction, the sample was purified via ultrafiltration. The AuNPs were dispersed in pure water to a final concentration of $0.05 \mathrm{mg} / \mathrm{mL}$ in $20 \mathrm{~mL}$, and a small amount of 
SDS was added to the suspension as a stabiliser. The coupling rate of the fluorescent peptide to the AuNPs was calculated based on the volume of the suspension and the amount of the fluorescent peptide.

\subsection{Probe characterisation}

The NP morphology and size were assessed using transmission electron microscopy (TEM) and dynamic light scattering (DLS). For sample preparation, AuNPs@PEG and AuNPs@PEG-Peptide-Cy5.5 in deionised water were placed on a 400-mesh copper grid and dried. TEM imaging was carried out using a FEI Tecnai F20 transmission electron microscope at an acceleration voltage of $200 \mathrm{kV}$. DLS measurements were performed using a Zetasizer Nano ZS90 (Malvern Instruments, Worcestershire, UK), and hydrodynamic sizes of samples were calculated. Fluorescence spectra were recorded using a fluorescence spectrometer (FLS980; Edinburgh, UK). NIR fluorescence imaging was performed using an IVIS Spectrum system (PerkinElmer).

\subsection{Animals}

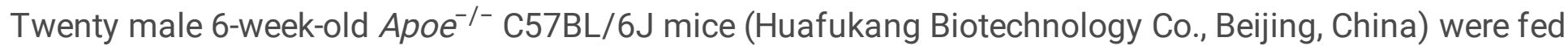
a standard laboratory chow diet (Cat \#1010009; Xietong Pharmaceuticals Co., Jiangsu, China) for 1 week. After acclimation, the mice were randomly allocated into a normal (control) group and a model group ( $\mathrm{n}=10$ each). The control group was fed the standard laboratory chow diet for 12 weeks. The model mice received a rigid, silicone, perivascular constrictive cuff placed around the right common carotid artery, as previously reported[26], and were fed a high-fat diet (HFD; 0.25\% cholesterol and 15\% cocoa butter). The animal handling and care and all procedures used in this study were performed in accordance with the regulations of the Animal Ethics Committee of Shandong University (IACUC Issue NO. DWLL-2021-001).

\subsection{Cell culture}

RAW 264.7 cells were obtained from the American Type Culture Collection and maintained in Dulbecco's modified Eagle's medium containing penicillin $(100 \mathrm{U} / \mathrm{mL})$, streptomycin $(100 \mu \mathrm{g} / \mathrm{mL})$, and $10 \%$ heatinactivated foetal calf serum (Gibco, Australia) at $37^{\circ} \mathrm{C}$ and $5 \% \mathrm{CO}_{2}$.

\subsection{Quantitative real-time polymerase chain reaction (qPCR)}

Total cellular RNA was extracted using the RNeasy mini kit (Qiagen) following the manufacturer's instructions. For animal samples, $100 \mathrm{mg}$ tissue was homogenized in $1 \mathrm{~mL}$ TRIzol reagent (Ambion), mixed vigorously in chloroform, and separated by centrifugation. Aqueous layer containing RNA was added to Qiagen RNEasy Plus column. cDNA was synthesised by reverse transcription of $1 \mu \mathrm{g}$ of RNA using the RevertAid first-strand cDNA synthesis kit (Thermo). qPCR was performed using a CFX Connect Real-Time PCR Detection System (BioRad) and a SYBR Green real-time PCR master mix (Toyobo), with fold-change quantified by $\triangle \Delta \mathrm{Ct}$. Gene expression levels were normalised to that of $\beta$-actin, used as an internal control. The primers used in this study were as follows: MMP-14, 5'-CCTCAAGTGGCAGCATAAT-3' and 5'-TGGAAACCCTCAGCAAAC-3'; MMP-2, 5'-CCCCGATGCTGATACTGA-3' and 5'- 
CACTGGCAAACCTTCACG-3'; CD68, 5'-ATGGGCTCGTATGATTGT-3' and 5'-CTTCTTAGGTTTCGGGTG-3'; CD206, 5'-GCAAGTGATTTGGAGGCT-3' and 5'-ATAGGAAACGGGAGAACC-3'; F4/80, 5'-

GTCAGATGATTCAGACGGAGTA-3' and 5'-TCACAGTGCCACCAACAA-3'; and $\beta$-actin, $5^{\prime}-$

CTGTGCCCATCTACGAGGGCTAT-3' and 5'-TTTGATGTCACGCACGATTTCC-3'.

\subsection{Western blotting}

RAW 264.7 cells or carotid tissues were homogenised and lysed in cold RIPA buffer (Beyotime, China) containing $1 \%$ phenylmethylsulphonyl fluoride. After lysate centrifugation at $12,000 \times g$ for 15 min at $4^{\circ} \mathrm{C}$, protein concentrations were quantified using a bicinchoninic acid protein assay kit (Beyotime). Equal amounts of protein from each sample were separated by $10 \%$ or $12 \%$ SDS-polyacrylamide gel electrophoresis, and separated proteins were transferred onto polyvinylidene fluoride membranes (Immobilon P; Millipore). The membranes were blocked with $10 \mathrm{mM}$ Tris-buffered saline (TBS) containing $5 \%$ skim milk at room temperature for $2 \mathrm{~h}$, followed by washing with TBS containing Tween 20 (TBST) for $5 \mathrm{~min}$. Afterwards, the membranes were incubated with the following primary antibodies: anti-MMP-14 (1:1,000, Cat \#ab51074; Abcam), anti-MMP-2 (1:1,000, Cat \#ab92536; Abcam), anti-CD68 (1:1,000, Cat \#ab213363; Abcam), anti-CD206 (1:1,000, Cat \#ab252921; Abcam), anti-F4/80 (1:1,000, Cat \#DF2789; Affinity), and anti- $\beta$-actin (1:5,000, Cat \#ab8227; Abcam) with $5 \%$ bovine serum albumin at $4^{\circ} \mathrm{C}$ overnight, followed by washing with TBST and incubation with a secondary antibody (1:5,000, Cat \#ab97080;

Abcam) for $1 \mathrm{~h}$ at room temperature. Immunoreactive bands were detected using an enhanced chemiluminescence kit (Merck Millipore) and a chemiluminescence system (Bio-Rad, USA) and processed using the Image Lab software.

\subsection{Histopathology}

Mice were sacrificed at 4, 8, and 12 weeks ( $n=3$ each) after cuff implantation to observe the formation of atherosclerotic plaques. Carotid artery samples were fixed with $10 \%$ formalin and embedded in paraffin at room temperature. The paraffin blocks were cut into 4- $\mu$ m-thick slices, which were stained with haematoxylin and eosin (H\&E) to calculate the areas of the vessel lumen and the inner and outer tunica media for each animal. Slices adjacent to the H\&E-stained slices were selected for immunohistochemical analysis for MMP-14, MMP-2, CD68, CD206, and F4/80. After deparaffinisation and rehydration, the slices were incubated with foetal bovine serum at $37^{\circ} \mathrm{C}$ for $30 \mathrm{~min}$, followed by incubation with primary rabbit and mouse monoclonal antibodies overnight at $4^{\circ} \mathrm{C}$, washing, and incubation with horseradish peroxidase-conjugated mouse anti-rabbit secondary antibodies. Finally, the slices were stained with 3, 3'diaminobenzidine (Solarbio, China), counterstained with haematoxylin, and scanned using a microscope (BX53; Olympus, Japan).

\subsection{Cytotoxicity assessment}

For the cell viability assay, cells were seeded at a density of $4 \times 10^{4}$ per well in 96 -well plates and incubated at $37^{\circ} \mathrm{C}$ in a $5 \% \mathrm{CO}_{2}$ incubator for $24 \mathrm{~h}$. Thereafter, the cells were treated with increasing concentrations ( 0 to $50 \mu \mathrm{g} / \mathrm{mL}$ ) of the fluorescent probe for $24 \mathrm{~h}$, and cell viability was evaluated using Cell Counting Kit-8 (CCK-8; Sigma-Aldrich, St. Louis, MO, USA). For apoptosis detection, cells were seeded 
in 6-well plates and cultured for $24 \mathrm{~h}$, followed by staining with annexin V-PI (BD Biosciences, Shanghai, China) and flow cytometry. To detect organ necrosis, model mice injected with saline and with probe were sacrificed $24 \mathrm{~h}$ post-injection and major organs were carefully harvested. All samples were stained with H\&E, placed on black paper, and imaged under a microscope.

\subsection{Immunofluorescence}

Raw 264.7 cells were seeded into an 8-well chamber slide (Ibidi, Germany) and then treated with or without $75 \mu \mathrm{g} / \mathrm{mL}$ oxidized low-density lipoprotein (ox-LDL) or GM6001. After $24 \mathrm{~h}$ of treatment, the cells were fixed with $4 \%$ paraformaldehyde and incubated with an MMP-14 antibody (1:500, Cat \#ab51074; Abcam) at $4^{\circ} \mathrm{C}$ overnight. The samples were then washed with cold phosphate-buffered saline (PBS) three times and incubated with an Alexa Fluor-conjugated secondary antibody at room temperature for 2 h. Afterwards, the cells were incubated with AuNPs@PEG-Peptide-Cy5.5 for another $1 \mathrm{~h}$, washed, and mounted with a glue solution containing 4', 6-diamidino-2-phenylindole (DAPI). Images were captured using a fluorescence microscope.

\subsection{In vivo imaging and ex vivo biodistribution studies}

In vivo images were acquired and analysed using the IVIS Spectrum system (PerkinElmer). All mice were shaved at the neck area, and those from the model group $(n=3)$ were administered AuNPs@PEG-PeptideCy 5.5 at a dose of $5 \mathrm{mg} / \mathrm{kg}$ via tail vein injection, while mice from the control group $(n=3)$ were injected saline. Imaging was performed at 10 and $30 \mathrm{~min}$ and 1, 2, 4, 8, 12, and $24 \mathrm{~h}$ after injection. During the injection and image acquisition process, the mice were anesthetised with $2.5 \%$ isoflurane in oxygen at a flow rate of $1.5 \mathrm{~L} / \mathrm{min}$. All images were normalised and analysed using the Maestro software. For quantitative comparison, regions of interest were drawn over the necks, and the average signal for each area was measured. For an ex vivo biodistribution study, mice were sacrificed at 24 and $48 \mathrm{~h}$ after injection of the probe. The major organs were carefully harvested and rinsed with PBS (pH 7.4), then placed on black paper, and immediately imaged using the IVIS Spectrum.

\subsection{Statistical analysis}

Continuous data were compared between the two groups using a $t$-test. A chi-squared or Fisher's exact test was used for categorical data. The nonparametric Mann-Whitney test was used for comparison of unpaired data. ANOVA test was used to compare means of three or more samples. Statistical significance was set at $p<0.05$. All statistical analyses were performed using GraphPad Prism version 8.0 (GraphPad Software, La Jolla, CA, USA) or the SPSS software version 21.0 (SPSS, Chicago, IL, USA) and presented as the mean \pm standard deviation.

\subsection{Role of the funding source}


Financial support was provided by the Transverse Research Project Foundation of China (No. 21520011691806). The funders had no role in the design and conduct of the study; collection, management, analysis and interpretation of the data; preparation, review or approval of the manuscript; and decision to submit the manuscript for publication. The corresponding authors have full access to all the data in the study and take responsibility for the integrity of the data and the accuracy of the data analysis.

\section{Results}

\subsection{Design and characterisation of AuNPs@PEG-Peptide- Cy5.5}

In this study, we first synthesised an MMP-14-specific and sensitive substrate peptide with a sequence of Gly-Arg-lle-Gly-Phe-Leu-Arg-Thr-Ala-Lys-Gly-Gly, which was then labelled at the N-terminus with a NIR fluorescent dye, Cy5.5. Subsequently, the Cy5.5-conjugated peptide was coupled to the surface of PEG 5000-wrapped AuNPs to construct a fluorescence resonance energy transfer system. Owing to the surface energy transfer properties[27], the fluorescence of Cy5.5 was quenched until the substrate peptide was degraded by the MMP-14 enzyme, which was highly expressed by M1 macrophages, as shown in Fig. 1. Based on the NIR fluorescence intensity, we could determine the expression of MMP-14 and inflammation progress and evaluate the possibility of plaque rupture.

A TEM image of AuNPs@PEG-Peptide-Cy5.5 is shown in Fig. 2a. The probe exhibited good dispersion in water, with an average particle diameter of $10 \mathrm{~nm}$. Compared with AuNPs@PEG, which had an average hydrodynamic diameter of $28 \cdot 76 \pm 7.37 \mathrm{~nm}$ (Fig. 2b), the probe displayed a similar or even higher monodispersity, with an average hydrodynamic diameter of 32.07 $\pm 6 \cdot 71 \mathrm{~nm}$ (Fig. 2c). The optical properties of the nanoprobe were determined by ultraviolet-visible (UV-Vis) spectroscopy and are displayed in Fig. 2d, which shows a peak at 520 nm, which is the typical absorbance of AuNPs@PEG, and peaks at 642 and $698 \mathrm{~nm}$, which are minimally different from the Cy5.5 absorbance at 634 and $677 \mathrm{~nm}$, indicating the successful conjugation of AuNPs and the Cy5.5-labelled peptide. The slight shifts in the absorption peaks of Cy5.5 were caused by its strong interaction with AuNPs. The peptide concentration in the purified supernatant of AuNPs@PEG-Peptide-Cy 5.5 was $31 \mathrm{mg} / \mathrm{mL}$ when the AuNPs@PEG concentration was $0.05 \mathrm{mg} / \mathrm{mL}$; therefore, we estimated that there were $1.77 \times 10^{2}$ molecules of the Cy5.5-labelled peptide per AuNP. The intensity of the AuNPs@PEG-Peptide-Cy5.5 peak, which appeared in an extremely narrow range, from 673 to $676 \mathrm{~nm}$ (Fig. 2e) was 56\% lower than that of the Cy5.5-labelled peptide, indicating an obvious quenching efficiency. To investigate the responsiveness of the probe to MMP-14, in vitro enzymatic assays were performed by incubating recombinant MMP-14 $(320 \mathrm{ng} / \mathrm{mL})$ and its inhibitor GM6001 with AuNPs@PEG-Peptide-Cy5.5 in PBS buffer (pH 7.4) at $37^{\circ} \mathrm{C}$ for $1 \mathrm{~h}$. As shown in Fig. $2 f$, a rapid fluorescence recovery of the nanoprobe was triggered by the addition of MMP-14, while the efficient fluorescence recovery of the nanoprobe was significantly prevented when the enzyme activity was inhibited by GM6001. To confirm that the NIR fluorescent signal of the probe was amplified as the 
MMP-14 concentration increased, an incubation was carried out with different concentrations $(0,10,20$, $40,80,160$, and $320 \mathrm{ng} / \mathrm{mL}$ ) of MMP-14. The NIR fluorescence emission signals of Cy5.5 were measured using a spectrofluorometer for $60 \mathrm{~min}$. As shown in Fig. 2g, the fluorescence was progressively intensified and positively linearly correlated with the concentrations of MMP-14 $\left(R^{2}=0 \cdot 94\right)$. To confirm the biological safety of the nanoprobe, in vitro cytotoxicity of AuNPs@PEG-Peptide-Cy5.5 was investigated using the CCK-8 assay (Fig. 2h) and flow cytometry analysis (Supplementary Fig. S1). Both assays demonstrated no significant cytotoxicity against RAW 264.7 cells after incubation with AuNPs@PEG-Peptide-Cy5.5 at different concentrations ( 0 to $50 \mu \mathrm{g} / \mathrm{mL}$ ) for $24 \mathrm{~h}$, and there were no significant differences in the populations of RAW 264.7 cells that underwent apoptosis and necrosis compared with those in the control without AuNPs@PEG-Peptide-Cy5.5 treatment.

\subsection{In vitro MMP-14 expression by activated macrophages and AuNPs@PEG-Peptide-Cy5.5 binding to cells}

The treatment of RAW 264.7 macrophages with ox-LDL $(80 \mu \mathrm{g} / \mathrm{mL})$ resulted in phenotypical characteristics of M1 and foamy macrophages, including upregulated expression of MMP-2 and inflammatory M1 markers, CD68 and F4/80 [28], and significant downregulation of the M2 marker CD206 [29]. Meanwhile, incubation with the MMP-14 inhibitor GM6001 for $24 \mathrm{~h}$ totally reversed the results (MMP14: $1.010 \pm 0.017$ versus $1.490 \pm 0.095$ versus $0.620 \pm 0.010, p<0.0001$; MMP-2: $0.993 \pm 0.040$ versus $1.640 \pm 0.095$ versus $0.677 \pm 0.067, p<0.0001 ; C D 68: 1.033 \pm 0.042$ versus $1.620 \pm 0.050$ versus $0.397 \pm$ $0.012, p<0.0001 ; F 4 / 80: 0.853 \pm 0.131$ versus $1.640 \pm 0.078$ versus $0.420 \pm 0.078, p<0.0001$; and CD206: $1.010 \pm 0.026$ versus $0.547 \pm 0.031$ versus $1.400 \pm 0.078, p<0.0001$, in the control, ox-LDL, and GM6001 treatment, respectively) (Fig. 3a and b). To validate the targeting effect of AuNPs@PEG-Peptide-Cy5.5, in vitro binding affinity experiments were first carried out using confocal laser scanning microscopy (Fig. 3c). Ox-LDL-stimulated macrophages showed remarkably stronger green fluorescence (MMP-14), whereas only faint fluorescence was detected in the inhibitor group. For the AuNPs@PEG-Peptide-Cy5.5stained groups (red), the fluorescence signal was clearly associated with the membrane, and the changing trend was consistent with that of antibody-labelled MMP-14.

\subsection{MMP-14 production in carotid samples and plaque formation}

To detect MMP-14 expression and visualise plaque formation, right carotid arteries were harvested every 4 weeks and analysed by QPCR and western blotting for inflammatory markers; by H\&E staining for morphological evidence; and by immunohistochemical staining for molecular signatures. As shown in Fig. $4 a$ and $b$, the results of qPCR and western blotting demonstrated gradually increased expression of MMP-14, MMP-2, CD-68, and F4/80 and reduced levels of CD206 in carotid samples collected from atherosclerotic model mice at $0,4,8$, and 12 weeks. Quantitative analysis (Fig. 4c) showed that the protein levels of MMP-14, MMP-2, CD68, and F4/80 were significantly upregulated (almost fivefold, $\mathrm{p}<$ 0.0001 ) in carotid samples from the model mice at 12 weeks compared with those at 0 weeks, while that of CD206 was largely reduced, indicating that the expression of MMP-14 increased with the enhancement 
of inflammatory activities of M1 macrophages. In addition, H\&E staining revealed that the enlargement of atherosclerotic plaques was accompanied by a thinning of fibrous caps and an increase of necrotic cores, which increased the likelihood of shoulder rupture, and immunohistochemical staining further confirmed increased levels of MMP-14, MMP-2, CD-68, and F4/80 and reduced deposition of CD206 (Fig. 4d). Thus, MMP-14 is a good marker that reflects the inflammatory responses of macrophages and the formation of rupture-prone plaques. We then simultaneously performed immunofluorescence staining using an MMP-14 antibody and nanoprobe staining of carotid samples and found that the stained areas overlapped. Furthermore, after preincubation of tissue sections with the MMP-14 inhibitor GM6001 for 30 min, the NIR fluorescent signal was significantly reduced, suggesting that AuNPs@PEG-Peptide-Cy5.5 had a good binding ability that could be used for MMP-14 detection (Fig. 4e).

\subsection{In vivo and ex vivo molecular imaging of carotid plaques}

To evaluate the feasibility of using AuNPs@PEG-Peptide-Cy5.5 for in vivo imaging of MMP-14 activity, the probe was intravenously administered to carotid atherosclerosis model mice and to control mice of the same age, sex, and background (Fig. 5a). Plaque formation was first assessed by microultrasound imaging to ensure that the carotid atherosclerosis model was successfully constructed (Supplementary Fig. S2). Next, the AuNPs@PEG-Peptide-Cy5.5 nanoprobe was injected into the tail vein of mice at a dose of $2.5 \mathrm{mg} / \mathrm{kg}$. In vivo fluorescence images were recorded over a period of $24 \mathrm{~h}$ at an excitation of $675 \mathrm{~nm}$ using an IVIS system. Representative images are shown in Fig. $5 \mathrm{a}$ at selected time points (10 and 30 min and 1, 2, 4, 8, 12, and $24 \mathrm{~h}$ ) after injection, with strong activation of the NIR fluorescence of AuNPs@PEGPeptide-Cy5.5 observed in the neck of the carotid atherosclerosis model mice. It should be noted that the probe showed a rapid, early onset of the activation (in less than $10 \mathrm{~min}$ ), and the fluorescence remained relatively steady for $2 \mathrm{~h}$, with a high average radiant efficiency compared with that in the control mice (Fig. 5b). After $2 \mathrm{~h}$, the fluorescent signal was quickly reduced and almost completely cleared after $12 \mathrm{~h}$. Additionally, ex vivo imaging showed stronger fluorescence signals in the right carotid arteries of the model mice than in those of the control mice treated with AuNPs@PEG-Peptide-Cy5.5 (Fig. 5c), which was consistent with the in vivo results shown in Fig. 5a. Collectively, these results strongly demonstrate that AuNPs@PEG-Peptide-Cy5.5 have a great potential as a NIR probe for sensitive detection of MMP-14 and for the diagnosis of the progression of atherosclerotic plaques.

\subsection{Ex vivo histological and biodistribution studies}

Ex vivo molecular imaging of various organs was performed at 24 and $48 \mathrm{~h}$ after in vivo imaging to detect biodistribution of the probe. As shown in Fig. $6 a$ and $b$, the biodistribution study indicated that AuNPs@PEG-Peptide-Cy5.5 was predominantly metabolised in the liver, kidney, and intestine. After 48 h, the average radiant efficiency declined by more than half, but that of the kidney was still higher than that of the other organs, indicating that the kidney was the main organ for the metabolism of the nanoprobe. H\&E staining images of various organs obtained from the control mice after intravenous injection of a PBS solution and from the model mice after AuNPs@PEG-Peptide-Cy 5.5 injection at a dose of $2.5 \mathrm{mg} / \mathrm{kg}$ showed no apparent abnormalities in the heart, pancreas, liver, kidney, muscle, lung, spleen, intestine, and 
brain (Fig. 6a). Therefore, our data confirmed that AuNPs@PEG-Peptide-Cy5.5 were notably effective in detecting MMP-14 activity and were not toxic to normal organs, suggesting that this probe can serve as an agent for rupture-prone plaque detection, with great clinical translational potential.

\section{Discussion}

In the present study, we confirmed that MMP-14, a cell membrane-anchoring enzyme, could serve as a biomarker of vulnerable plaques. The data also demonstrated that the MMP-14 substrate-based fluorescent nanoprobe AuNPs@PEG-Peptide-Cy5.5, which showed an intense fluorescence signal after activation and good biocompatibility, could be applied to screen for and monitor plaque progression in vivo.

Satisfactory imaging techniques are urgently needed to detect vulnerable plaques, which are mediated by inflammatory activities and cause lethal cerebrovascular events [30,31]. In recent years, molecular targetbased imaging of the degree of plaque inflammation has been considered a promising approach for identifying rupture-prone plaques [32, 33]. Many molecular biomarkers, such as MMPs [34], hypoxiarelated factors, and vascular cell adhesion molecules [35], have been proposed. Of these, MMPs are particularly attractive, as they are proteinases that directly degrade the matrix and erode the fibrous cap and are involved in a series of other regulatory mechanisms that promote plaque instability. Seifert et al. [34] implanted a tapered cuff around the right common carotid artery in $A p o e^{-/-}$mice to induce a model of atherosclerosis and found that the MMP-2 and MMP-9 activities were significantly higher in upstream, low shear stress-induced unstable atherosclerotic plaques than in downstream, more stable plaque phenotypes. However, many fluorogenic probes targeting MMP-2 and MMP-9 have been demonstrated to have limitations in in vivo applications. One important reason is that both MMP-2 and MMP-9 are extracellularly secreted, soluble types of MMPs. They are abundantly secreted into the bloodstream, providing high background signals, and probes are easily washed away, which thus lowers the imaging quality $[36,37]$. To compensate for these drawbacks, many reported MMP-2 and MMP-9 probes were designed to carry another recognition sequence to anchor these probes to the extracellular membrane of specific cells, which increased the technical difficulty of probe synthesis, while the effects might not have been as expected [38]. Unlike extracellular MMPs, membrane-type (MT)-MMPs are tethered to the plasma membrane via either a glycosylphosphatidylinositol linkage or a transmembrane domain [39]. Moreover, MMP-14, also known as MT1-MMP, is directly linked to the pathogenesis of plaque vulnerability, as MMP14 has been shown to convert MMP-2 and MMP-9 proenzymes into their active forms and to act as an attractant to facilitate monocyte/macrophage infiltration into sites of experimentally induced inflammation and established atherosclerotic lesions $[5,40]$. To detect cardiac ischemia/reperfusion injury, van Duijnhoven et al. [41] developed a series of activatable cell-penetrating peptides (ACPPs) that were sensitive to MMP-2 and MMP-9 (ACPP-2/9) or to MMP-14 (ACPP-B). Although both ACPPs successfully detected regions of an infarcted myocardium in mouse models, the ACPP-2/9 probe showed a considerable degree of activation in all tissues, while ACPP-B was found to be tissue specific. Therefore, MMP-14 is more suitable than MMP-2 and MMP-9 as a target for plaque inflammation imaging. 
In this study, the core sequence, Arg-Ile-Gly-Phe-Leu-Arg, of the peptide was adopted from previous reports. This sequence has been verified to have a high specificity and selectivity for MT-MMPs, including MT1-MMP (MMP-14), MT2-MMP (MMP-15), and MT3-MMP (MMP-16), as well as for MMP-2 and MMP-9 $[25,42]$. Next, a NIR fluorescent dye, Cy5.5, and PEG 5000-wrapped AuNPs were conjugated to the N-and C-termini of the peptide, respectively. Characterisation tests demonstrated that AuNPs@PEG-PeptideCy5.5 had a small hydrated size and homogeneous size distribution, which would allow the NPs to easily penetrate into a plaque through a damaged endothelium or the vasa vasorum. The fluorescence intensity of AuNPs@PEG-Peptide-Cy5.5 was negligible in a static state because the signal could only be detected in a narrow range; however, after the activation, the signal could be amplified up to several folds, depending on the MMP-14 concentration. The biosafety assessment of AuNPs@PEG-Peptide-Cy5.5 showed its low cytotoxicity and no significant injuries to organs such as the lung, heart, liver, and kidney.

MMPs not only participate in the onset and progression of atherosclerosis but also reflect plaque vulnerability [43], and macrophages are the most important source of MMPs in atherosclerosis. Hence, in vitro biological evaluation of the MMP-14-targeting fluorescent nanoprobe was performed using the macrophage cell line RAW 264.7. The results showed that MMP-14 was highly expressed in ox-LDLinduced foamy macrophages, at a level of $5 \cdot 2$-fold higher than that in normal macrophages. To verify the association of MMP-14 expression with inflammatory macrophages, we determined changes in the M1 macrophage markers CD68 and F4/80 [44] and the classical M2 macrophage marker CD206 [45] and confirmed that CD68 and F4/80 expression was significantly upregulated and that of CD206 was largely suppressed in ox-LDL-induced macrophages. These trends were reversed by the treatment with the nonselective MMP inhibitor GM6001 [46], which confirmed that MMP-14 could potentially serve as a marker for macrophage inflammatory activities. Consistent with the in vitro data, the expression of MMP14 increased and the hyperactivity of macrophages was aggravated with plaque progression in the murine model of atherosclerosis. Cell and tissue fluorescence imaging suggested a targeting ability of AuNPs@PEG-Peptide-Cy5.5 towards MMP-14, as the area of nanoprobe binding overlapped with that of MMP-14 expression. In vivo, a distinctive fluorescence signal reached a peak value within $10 \mathrm{~min}$ after probe injection and disappeared at $12 \mathrm{~h}$ post-injection. To eliminate interference from other tissues and confirm that the fluorescent signal was indeed associated with the right carotid artery, wherein plaques were induced, the right carotid artery was immediately isolated after AuNPs@PEG-Peptide-Cy5.5 injection, and ex vivo studies showed that the probe was preferentially accumulated in the plaque on the right artery wall. Biodistribution studies indicated that this nanoprobe had a short circulation time and could be rapidly cleared through the liver, kidney, and intestine, which lowered the risk of possible injury to organs and tissues.

There are some limitations to this study. First, the AuNPs@PEG-Peptide-Cy5.5 nanoprobe was used in a single imaging mode and only for the diagnosis of vulnerable plaques. Meanwhile, recently developed probes tend to have dual imaging modalities or be functional in both diagnosis and treatment, such as dual-functional, fluorescent-radiolabelled, composite imaging agents [47, 48], which can simultaneously detect PET and NIR fluorescence signals, or photodynamic/photothermal therapeutic drugs, which are not only used for imaging but also provide thermotherapy to kill cancer cells $[49,50]$. Second, the 
implantation of the silicone cuff might have induced perivascular inflammation, thus causing falsepositive results, such as non-atherosclerosis-related background signals for imaging. More importantly, human vessels cannot be fully mimicked by mouse vessels, in which cardio-cerebrovascular events are rare, for unknown reasons. Third, in vitro fluorescent imaging of plaque samples showed that the internal elastic membrane could also be stained by the nanoprobe, and further studies are needed to elucidate the mechanism of this off-target effect and make improvements.

\section{Conclusions}

In summary, in this study, we investigated and validated the mechanism by which MMP-14, a promising biomarker of M1 macrophages, contributes to the progression and rupture of atherosclerotic plaques. Then, we successfully developed an MMP-14-specific, enzyme-activated NIR nanoprobe, AuNPs@PEGPeptide-Cy5.5, for rapid and non-destructive in vivo visualisation of vulnerable atherosclerotic plaques, which would be a great help to make clinical decision.

\section{Abbreviations}

Matrix Metalloproteinase (MMP); Cyanine (Cy); Gold Nanoparticles (AuNPs); Polyethylene Glycol (PEG) ; Positron Emission Tomography (PET); Near-infrared (NIR); Transmission Electron Microscopy (TEM); Cell Counting Kit-8 (CCK-8); Quantitative Real-time Polymerase Chain Reaction (qPCR) ; Oxidized Low-density Lipoprotein (ox-LDL); 4', 6-Diamidino-2-Phenylindole (DAPI); Fluorescence Resonance Energy Transfer (FRET); Activatable Cell-penetrating Peptides (ACPPs); Membrane-type Matrix Metalloproteinase MTMMPs

\section{Declarations}

\section{Ethical approval and consent to participate}

The research study was approved by the Animal Ethics Committee of Qilu Hospital of Shandong University. Informed consent was based on the Declaration of Helsinki.

\section{Consent for publication}

Not applicable.

\section{Availability of data and materials}

The data that support the findings of this study are available in the manuscript and from the corresponding author upon request (drwangdonghai@sdu.edu.cn).

\section{Competing interests}

The authors have no conflicts of interest to declare. 


\section{Funding}

Financial support was provided by the Transverse Research Project Foundation of China (No. 21520011691806). The funders had no role in the design and conduct of the study; collection, management, analysis and interpretation of the data; preparation, review or approval of the manuscript; and decision to submit the manuscript for publication. The corresponding authors have full access to all the data in the study and take responsibility for the integrity of the data and the accuracy of the data analysis.

\section{Author contributions}

Conceptualization and study design: D.W. and M.H.; Literature search and data collection: K.L, T.S., and H.X.; Data interpretation: F.W., Y.H.; Figures: M.H.; Writing - original draft: M.H.; Writing - review \& editing: D.W., K.L, and H.X.; Project supervision: D.W.; Funding acquisition: D.W. All authors have read and approved the final version of this manuscript.

\section{Acknowledgements}

This work was supported by a grant from the Transverse Research Project Foundation of China (No. 21520011691806). We wish to thank all past and present members of our laboratories for critically reading of our manuscript.

\section{References}

1. Gokaldas, R., et al., Carotid stenosis: from diagnosis to management, where do we stand? Curr Atheroscler Rep, 2015. 17(2): p. 480.

2. Zhu, Y., et al., Research Progress on the Relationship between Atherosclerosis and Inflammation. Biomolecules, 2018. 8(3).

3. Newby, A.C., et al., Vulnerable atherosclerotic plaque metalloproteinases and foam cell phenotypes. Thromb Haemost., 2009. 101: p. 1006-1011.

4. Mushenkova, N.V., et al., Current Advances in the Diagnostic Imaging of Atherosclerosis: Insights into the Pathophysiology of Vulnerable Plaque. Int J Mol Sci, 2020. 21(8).

5. Di Gregoli, K., et al., Differential effects of tissue inhibitor of metalloproteinase (TIMP)-1 and TIMP-2 on atherosclerosis and monocyte/macrophage invasion. Cardiovasc Res, 2016. 109(2): p. 318-30.

6. Newby, A.C., Metalloproteinase production from macrophages - a perfect storm leading to atherosclerotic plaque rupture and myocardial infarction. Exp Physiol, 2016. 101(11): p. 1327-1337.

7. Brown, B.A., H. Williams, and S.J. George, Chapter Six - Evidence for the Involvement of MatrixDegrading Metalloproteinases (MMPs) in Atherosclerosis, in Progress in Molecular Biology and Translational Science, R.A. Khalil, Editor. 2017, Academic Press. p. 197-237.

8. Guo, Z.Y., et al., Specific matrix metalloproteinases and calcification factors are associated with the vulnerability of human carotid plaque. Exp Ther Med, 2018. 16(3): p. 2071-2079. 
9. Sato, H., et al., A matrix metalloproteinase expressed on the surface of invasive tumour cells. Nature, 1994. 370(6484): p. 61-5.

10. Hernandez-Barrantes, S., et al., Regulation of membrane type-matrix metalloproteinases. Semin Cancer Biol, 2002. 12(2): p. 131-8.

11. Yan, D., et al., MMP-14 promotes VSMC migration via up-regulating CD44 expression in cardiac allograft vasculopathy. Pathol Res Pract, 2016. 212(12): p. 1119-1125.

12. Wang, J., et al., p85alpha Inactivates MMP-2 and Suppresses Bladder Cancer Invasion by Inhibiting MMP-14 Transcription and TIMP-2 Degradation. Neoplasia, 2019. 21(9): p. 908-920.

13. Schneider, F., et al., Matrix-metalloproteinase-14 deficiency in bone-marrow-derived cells promotes collagen accumulation in mouse atherosclerotic plaques. Circulation, 2008. 117(7): p. 931-9.

14. Kuge, Y., et al., Distribution Profiles of Membrane Type-1 Matrix Metalloproteinase (MT1-MMP), Matrix Metalloproteinase-2 (MMP-2) and Cyclooxygenase-2 (COX-2) in Rabbit Atherosclerosis: Comparison with Plaque Instability Analysis. Biological and Pharmaceutical Bulletin, 2007. 30(9): p. 1634-1640.

15. Johnson, J.L., et al., Low Tissue Inhibitor of Metalloproteinases 3 and High Matrix Metalloproteinase 14 Levels Defines a Subpopulation of Highly Invasive Foam-Cell Macrophages. Arterioscler Thromb Vasc Biol, 2008. 28: p. 1647-1653.

16. Jaffer, F.A., P. Libby, and R. Weissleder, Molecular imaging of cardiovascular disease. Circulation, 2007. 116(9): p. 1052-61.

17. Khraishah, H. and F.A. Jaffer, Intravascular Molecular Imaging: Near-Infrared Fluorescence as a New Frontier. Front Cardiovasc Med, 2020. 7: p. 587100.

18. Kuge, Y., et al., Imaging with radiolabelled anti-membrane type 1 matrix metalloproteinase (MT1MMP) antibody: potentials for characterizing atherosclerotic plaques. Eur J Nucl Med Mol Imaging, 2010. 37(11): p. 2093-104.

19. Tsimikas, S., Noninvasive imaging of oxidized Low-Density Lipoprotein in atherosclerotic plaques with tagged oxidation-specific antibodies. The American Journal of Cardiology, 2002. 90(10, Supplement 3): p. L22-L27.

20. Collet, C., et al., Reviewing imaging modalities for the assessment of plaque erosion. Atherosclerosis, 2021. 318: p. 52-59.

21. Kosuge, $\mathrm{H}$., et al., Near infrared imaging and photothermal ablation of vascular inflammation using single-walled carbon nanotubes. J Am Heart Assoc, 2012. 1(6): p. e002568.

22. Pala, R., et al., Nanomaterials as Novel Cardiovascular Theranostics. Pharmaceutics, 2021. 13(3).

23. Yi, X., et al., Near-infrared fluorescent probes in cancer imaging and therapy: an emerging field. Int $\mathrm{J}$ Nanomedicine, 2014. 9: p. 1347-65.

24. Shi, H., et al., Real-time monitoring of cell apoptosis and drug screening using fluorescent light-up probe with aggregation-induced emission characteristics. J Am Chem Soc, 2012. 134(43): p. 1797281. 
25. Zhu, L., et al., In vivo optical imaging of membrane-type matrix metalloproteinase (MT-MMP) activity. Mol Pharm, 2011. 8(6): p. 2331-8.

26. Ni, M., et al., Micro-ultrasound imaging assessment of carotid plaque characteristics in apolipoprotein-E knockout mice. Atherosclerosis, 2008. 197(1): p. 64-71.

27. Wu, L., et al., Förster resonance energy transfer (FRET)-based small-molecule sensors and imaging agents. Chem Soc Rev, 2020. 49(15): p. 5110-5139.

28. Ismahil, M.A., et al., Remodeling of the mononuclear phagocyte network underlies chronic inflammation and disease progression in heart failure: critical importance of the cardiosplenic axis. Circ Res, 2014. 114(2): p. 266-82.

29. Lv, R., Q. Bao, and Y. Li, Regulation of M1-type and M2-type macrophage polarization in RAW264.7 cells by Galectin-9. Mol Med Rep, 2017. 16(6): p. 9111-9119.

30. Gonzalez, L. and B.L. Trigatti, Macrophage Apoptosis and Necrotic Core Development in Atherosclerosis: A Rapidly Advancing Field with Clinical Relevance to Imaging and Therapy. Can J Cardiol, 2017. 33(3): p. 303-312.

31. Anwaier, G., et al., A review of molecular imaging of atherosclerosis and the potential application of dendrimer in imaging of plaque. Int J Nanomedicine, 2017. 12: p. 7681-7693.

32. Wang, J., et al., Scavenger receptor-Al-targeted ultrasmall gold nanoclusters facilitate in vivo MR and ex vivo fluorescence dual-modality visualization of vulnerable atherosclerotic plaques.

Nanomedicine, 2019. 19: p. 81-94.

33. Wang, Y., et al., Optical/MRI dual-modality imaging of M1 macrophage polarization in atherosclerotic plaque with MARCO-targeted upconversion luminescence probe. Biomaterials, 2019. 219: p. 119378.

34. Seifert, R., et al., Molecular imaging of MMP activity discriminates unstable from stable plaque phenotypes in shear-stress induced murine atherosclerosis. PLoS One, 2018. 13(10): p. e0204305.

35. Chan, J.M.S., et al., Imaging vulnerable plaques by targeting inflammation in atherosclerosis using fluorescent-labeled dual-ligand microparticles of iron oxide and magnetic resonance imaging. J Vasc Surg, 2018. 67(5): p. 1571-1583 e3.

36. Sun, L., et al., MMP-2-responsive fluorescent nanoprobes for enhanced selectivity of tumor cell uptake and imaging. Biomater Sci, 2018. 6(10): p. 2619-2626.

37. Ji, X., et al., MT1-MMP activatable fluorogenic probes with enhanced specificity via high-affinity peptide conjugation for tumor imaging. Biomater Sci, 2020. 8(8): p. 2308-2317.

38. Yin, L., et al., Rational Design and Synthesis of a Metalloproteinase-Activatable Probe for DualModality Imaging of Metastatic Lymph Nodes in Vivo. The Journal of Organic Chemistry, 2019. 84(10): p. 6126-6133.

39. Shimokawa, H., Bone-marrow-derived matrix metalloproteinase-14: a novel target for plaque stability. Circulation, 2008. 117(7): p. 863-5.

40. Karamanos, N.K., et al., Matrix modeling and remodeling: A biological interplay regulating tissue homeostasis and diseases. Matrix Biol, 2019. 75-76: p. 1-11. 
41. van Duijnhoven, S.M., et al., Development of Radiolabeled Membrane Type-1 Matrix Metalloproteinase Activatable Cell Penetrating Peptide Imaging Probes. Molecules, 2015. 20(7): p. 12076-92.

42. Kridel, S.J., et al., A unique substrate binding mode discriminates membrane type-1 matrix metalloproteinase from other matrix metalloproteinases. J Biol Chem, 2002. 277(26): p. 23788-93.

43. Johnson, J.L., Metalloproteinases in atherosclerosis. Eur J Pharmacol, 2017. 816: p. 93-106.

44. Yang, S., et al., Macrophage polarization in atherosclerosis. Clin Chim Acta, 2020. 501: p. 142-146.

45. Arnold, K.A., et al., Monocyte and macrophage subtypes as paired cell biomarkers for coronary artery disease. Exp Physiol, 2019. 104(9): p. 1343-1352.

46. Han, K.Y., J.H. Chang, and D.T. Azar, MMP14-Containing Exosomes Cleave VEGFR1 and Promote VEGFA-Induced Migration and Proliferation of Vascular Endothelial Cells. Invest Ophthalmol Vis Sci, 2019. 60(6): p. 2321-2329.

47. Houghton, J.L., et al., Site-specifically labeled CA19.9-targeted immunoconjugates for the PET, NIRF, and multimodal PET/NIRF imaging of pancreatic cancer. Proc Natl Acad Sci U S A, 2015. 112(52): $p$. 15850-5.

48. Seo, J.W., et al., (64)Cu-labeled LyP-1-dendrimer for PET-CT imaging of atherosclerotic plaque. Bioconjug Chem, 2014. 25(2): p. 231-9.

49. Gao, S., et al., Hybrid graphene/Au activatable theranostic agent for multimodalities imaging guided enhanced photothermal therapy. Biomaterials, 2016. 79: p. 36-45.

50. Chen, $\mathrm{H}$., et al., Cartilage-targeting and dual MMP-13/pH responsive theranostic nanoprobes for osteoarthritis imaging and precision therapy. Biomaterials, 2019. 225: p. 119520.

\section{Figures}




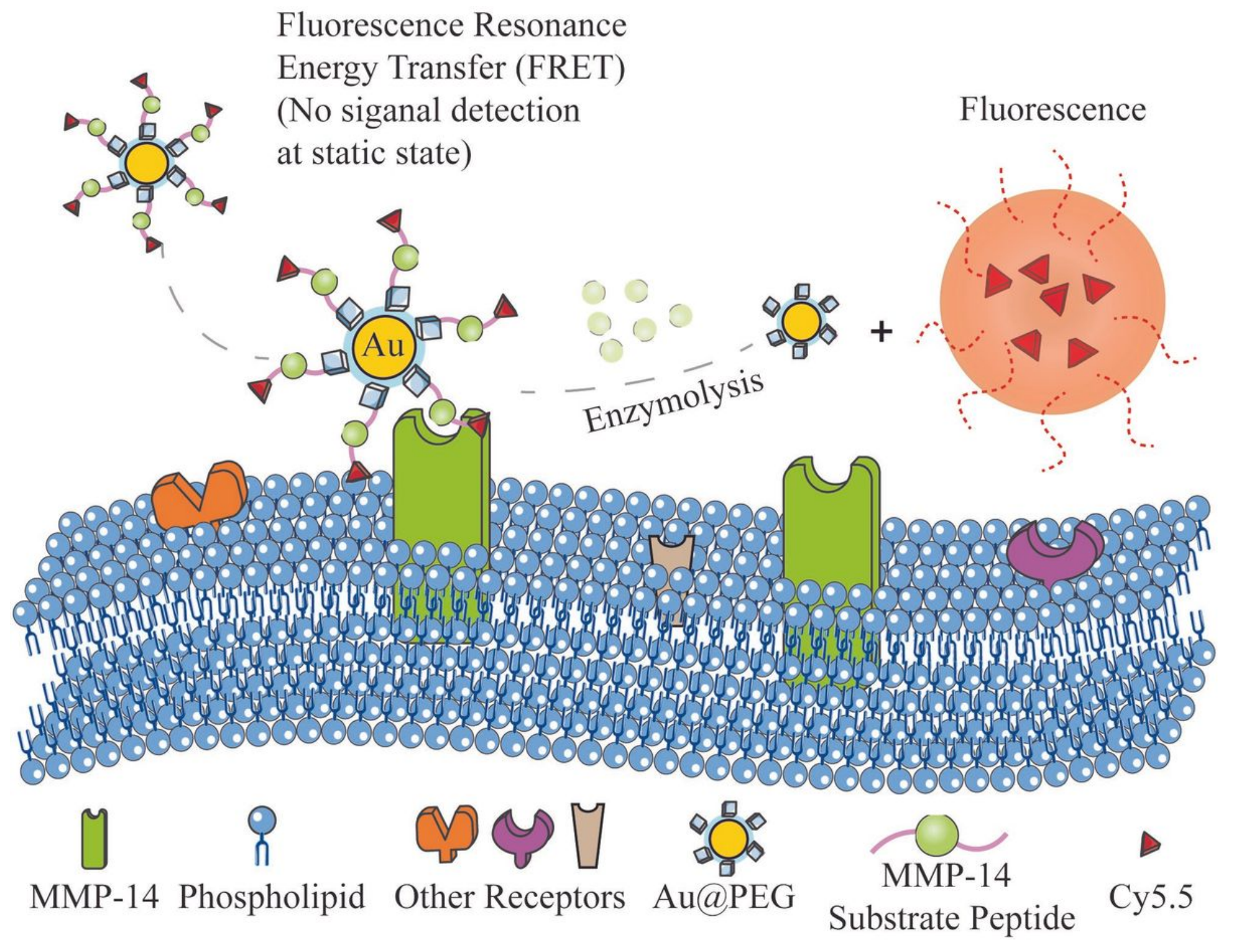

Figure 1

Schematic illustration of AuNPs@PEG-Peptide-Cy5.5 as a NIR fluorescence imaging probe. The probe includes three parts: fluorescence dye Cy5.5, MMP-14 substrate (peptide as a linker), and PEG5000 wrapped nanogold particles (AuNPs). Cy5.5 is covalently linked to the MMP-14 substrate and is incorporated into the surface of AuNPs, for which the fluorescence will be quenched owing to surface energy transfer properties. However, upon proteolytic cleavage with MMP-14, Cy5.5 could emit strong near-infrared fluorescence because the quenching effect was reduced as the distance increased. 
A

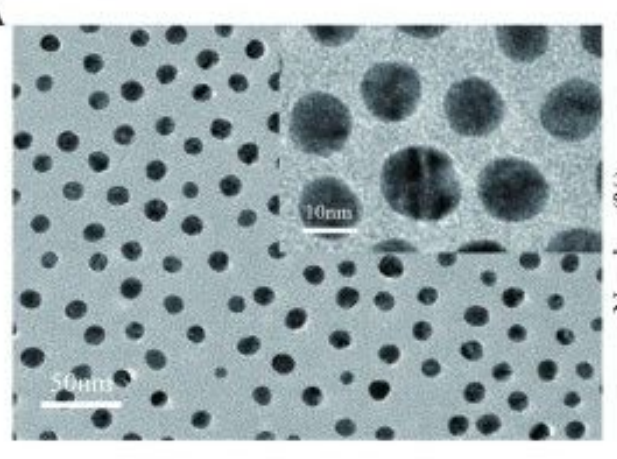

D

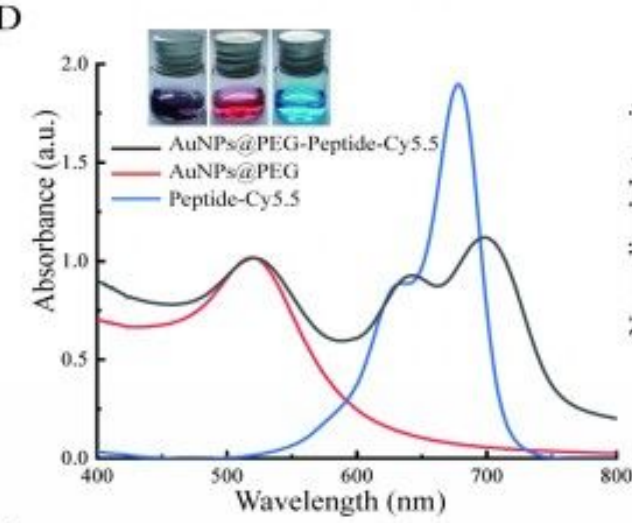

G

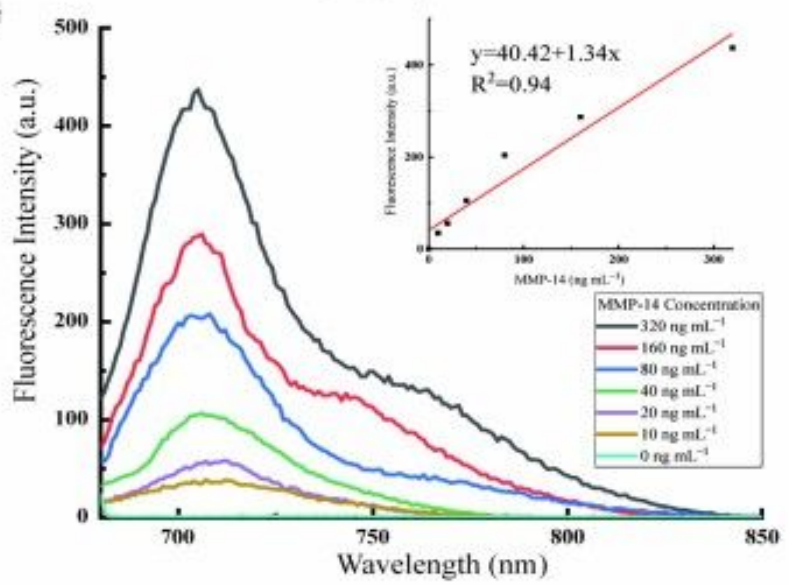

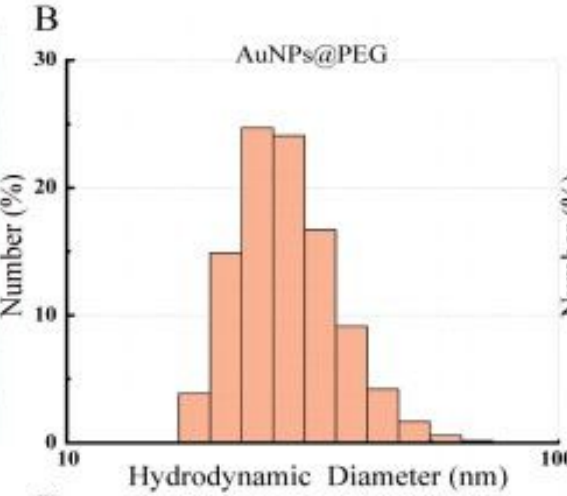

C

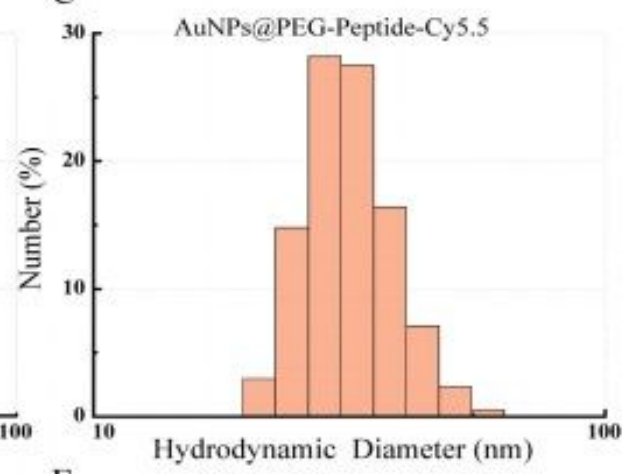

E - AuNPsäPEG-Peptide-Cy 5.5
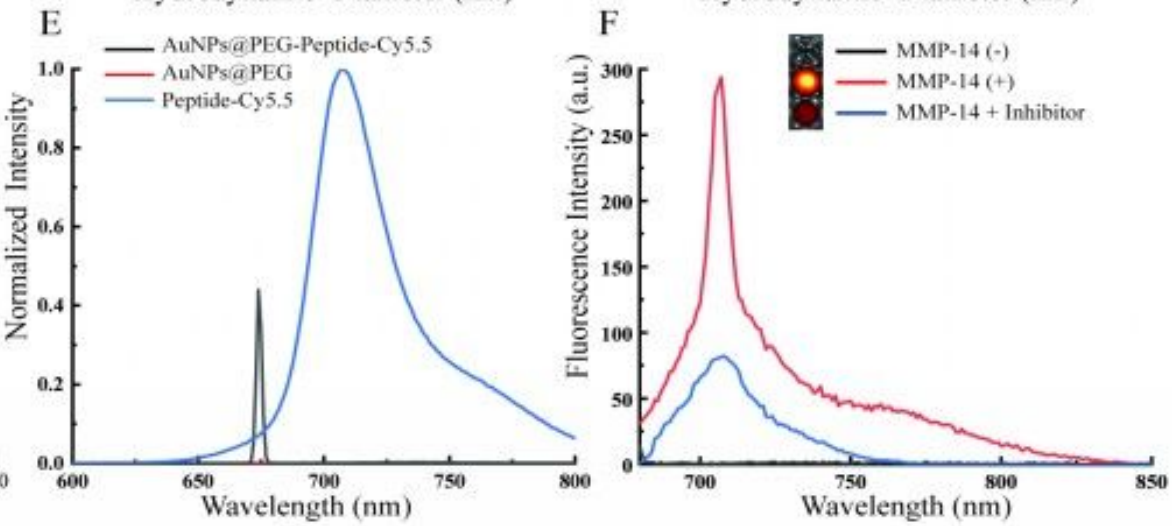

$\mathrm{H}$

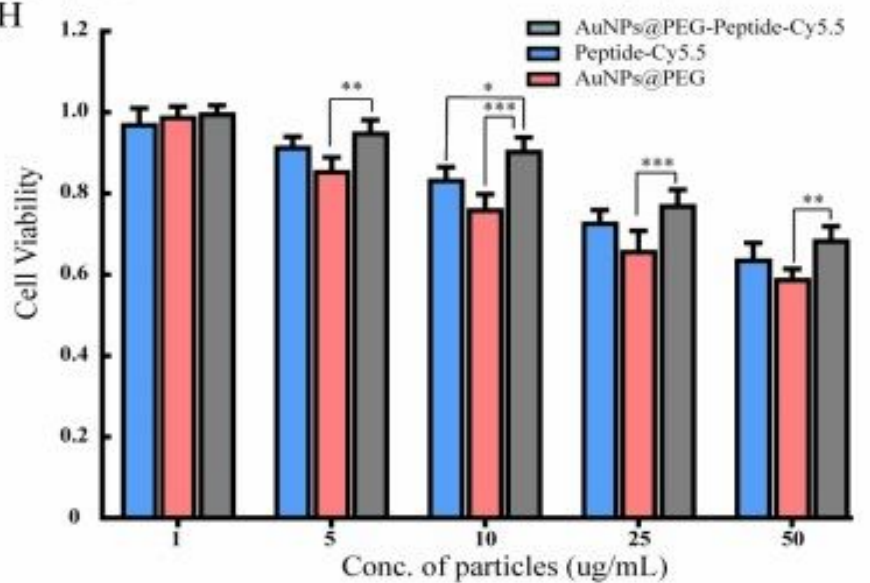

Figure 2

Characterisation of AuNPs@PEG-Peptide-Cy5.5. (a) TEM image of synthesised AuNPs@PEG-PeptideCy5.5, with an average particle size of approximately $10 \mathrm{~nm}$. (b and c) Particle size distributions of AuNPs@PEG (b) and AuNPs@PEG-Peptide-Cy5.5 (c), assessed by DLS. (d) UV-Vis spectra of AuNPs@PEG-Peptide-Cy5.5,AuNPs@PEG, and the Cy5.5-labelled peptide in aqueous solutions. Inset: optical images of the above solutions. (e) Fluorescence spectra of AuNPs@PEG-Peptide-Cy5.5, AuNPs@PEG, and the Cy5.5-labelled peptide. (f) Fluorescence spectra of AuNPs@PEG-Peptide-Cy5.5 after incubation with and without MMP-14 (320 ng/mL) and its inhibitor GM6001 (100 $\mu \mathrm{M})$ at $37^{\circ} \mathrm{C}$ for $1 \mathrm{~h}$ in PBS (pH 7-4). Inset: fluorescence images of the above solutions recorded at an excitation of $646 \mathrm{~nm}$. (g) Fluorescence recovery spectra of AuNPs@PEG-Peptide-Cy5.5 at the indicated concentrations of MMP-14. Inset: correlation between fluorescence intensity and the concentration of MMP-14. (h) Viability of RAW 264.7 cells incubated with different concentrations of AuNPs@PEG-Peptide-Cy5.5, AuNPs@PEG, and the 
Cy5.5-labelled peptide for $24 \mathrm{~h}$. Data analyzed by two-way ANOVA with post-hoc Sidak's multiple comparisons for differences within groups at each concentration. Error bars indicate standard deviation $(n=3)\left({ }^{*} p<0.05,{ }^{*} p<0.01, * * * p<0.001\right)$.

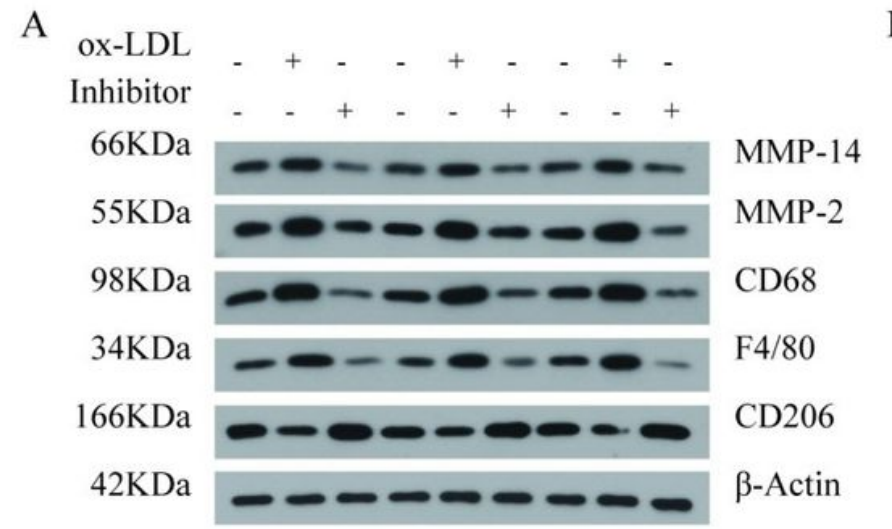

C
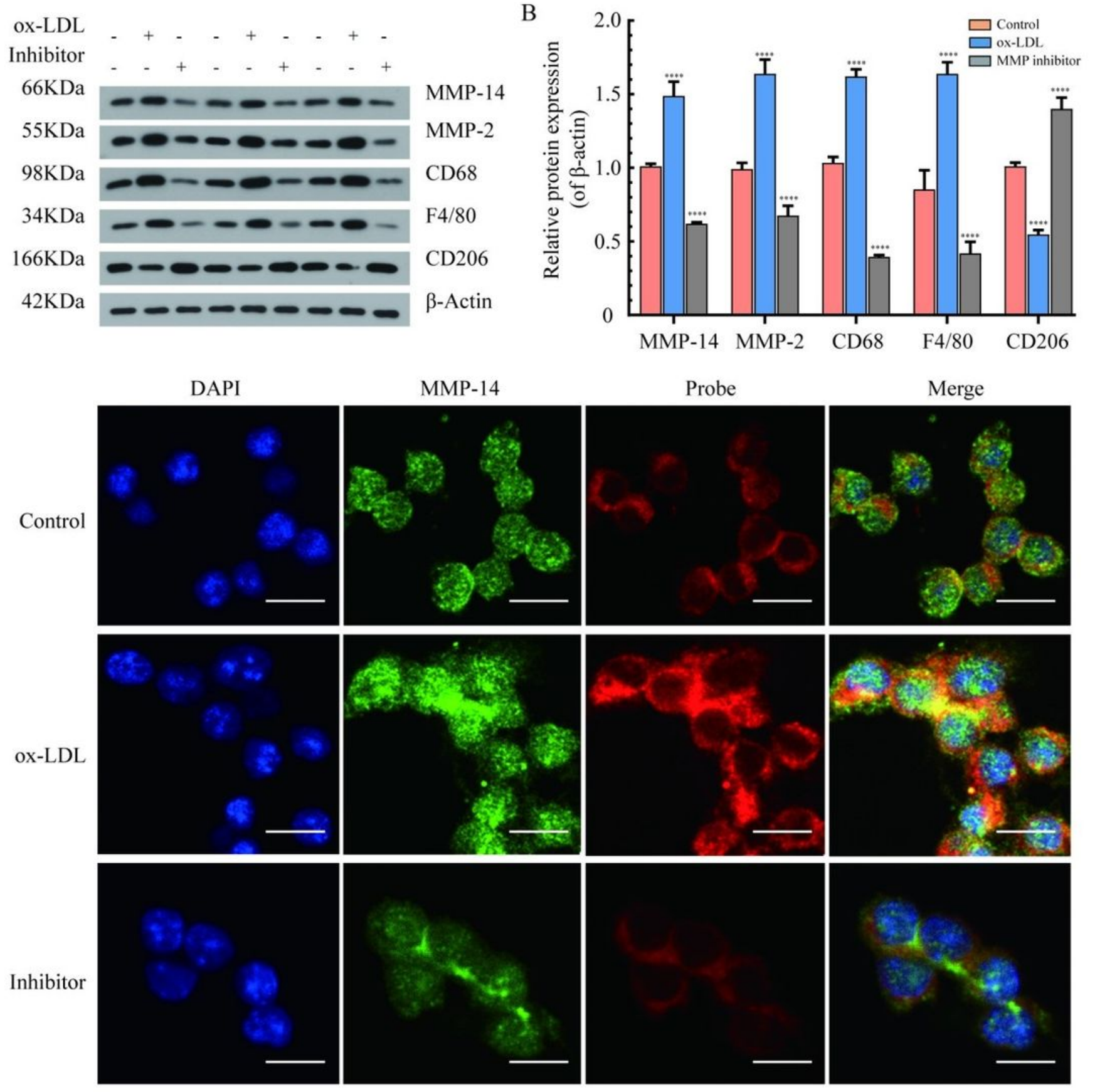

\section{Figure 3}

In vitro MMP-14 expression in ox-LDL-induced macrophages and AuNPs@PEG-Peptide-Cy5.5 binding ability (a) Western blots of MMP-14, MMP-2, CD68, F4/80, and CD206 expression in macrophages treated with ox-LDL or the nonspecific MMP inhibitor GM6001. (b) Quantitative analysis of MMP-14, MMP-2, CD68, F4/80, and CD206 levels normalised to those of $\beta$-actin. Data presented as mean \pm S.D. for $n=3$ per group, with ${ }^{\star \star * \star} p<0.0001$ by two-way ANOVA with post-hoc Sidak's multiple comparisons for 
differences within groups for different treatment. (c) MMP-14 immunofluorescence (green) and probe staining (red) of RAW 264.7 macrophages incubated with or without ox-LDL induction and GM6001 treatment.

A

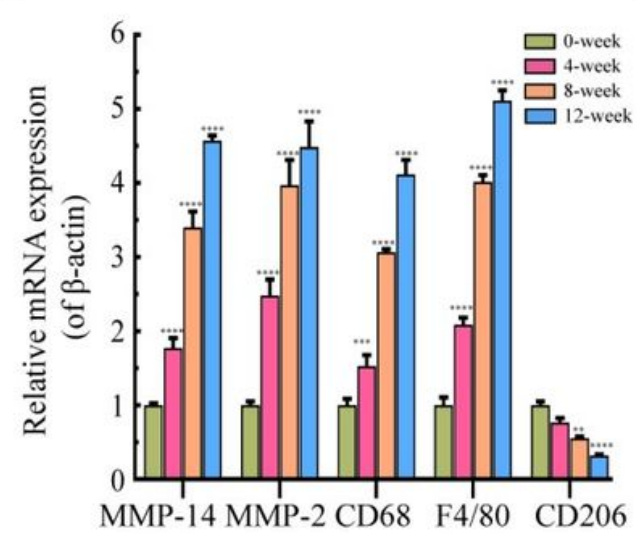

B

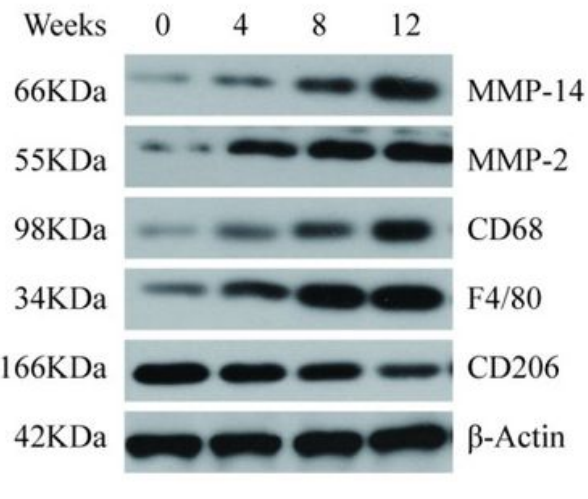

$\mathrm{D}$

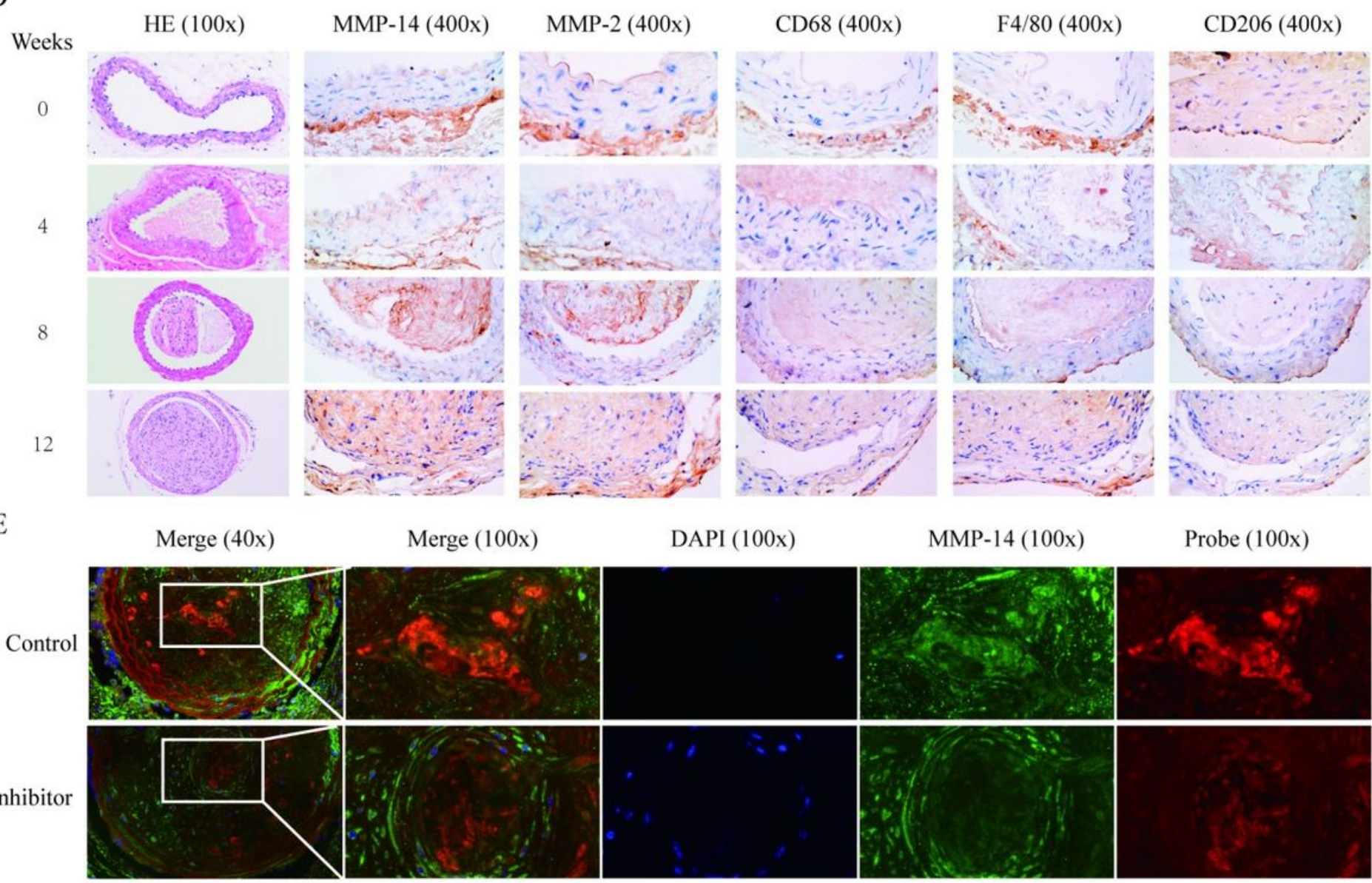

\section{Figure 4}

Expression of MMP-14 and related inflammatory markers within plaques. (a) mRNA and (b) protein expression of MMP-14, MMP-2, CD68, F4/80, and CD206 in the right carotid artery of mice at $0,4,8$, and 12 weeks. (c) Quantitative analysis of the western blot data in (b). Data presented as mean \pm S.D. $(n=3$ per group), with ${ }^{* *} p<0.01,{ }^{* *} \mathrm{p}<0.001,{ }^{* * *} \mathrm{p}<0.0001$ by two-way ANOVA with post-hoc Sidak's multiple 
comparisons for differences within groups at different time point. (d) H\&E-stained carotid arteries, showing the intima-medial thickness, and carotid arteries immunostained for MMP-14, MMP-2, CD68, F4/80, and CD206. Apoe-/- mice were fed an HFD for 0, 4, 8, and 12 weeks. (E) Immunofluorescence (green) and probe staining (red) of carotid artery tissues with or without GM6001 treatment.
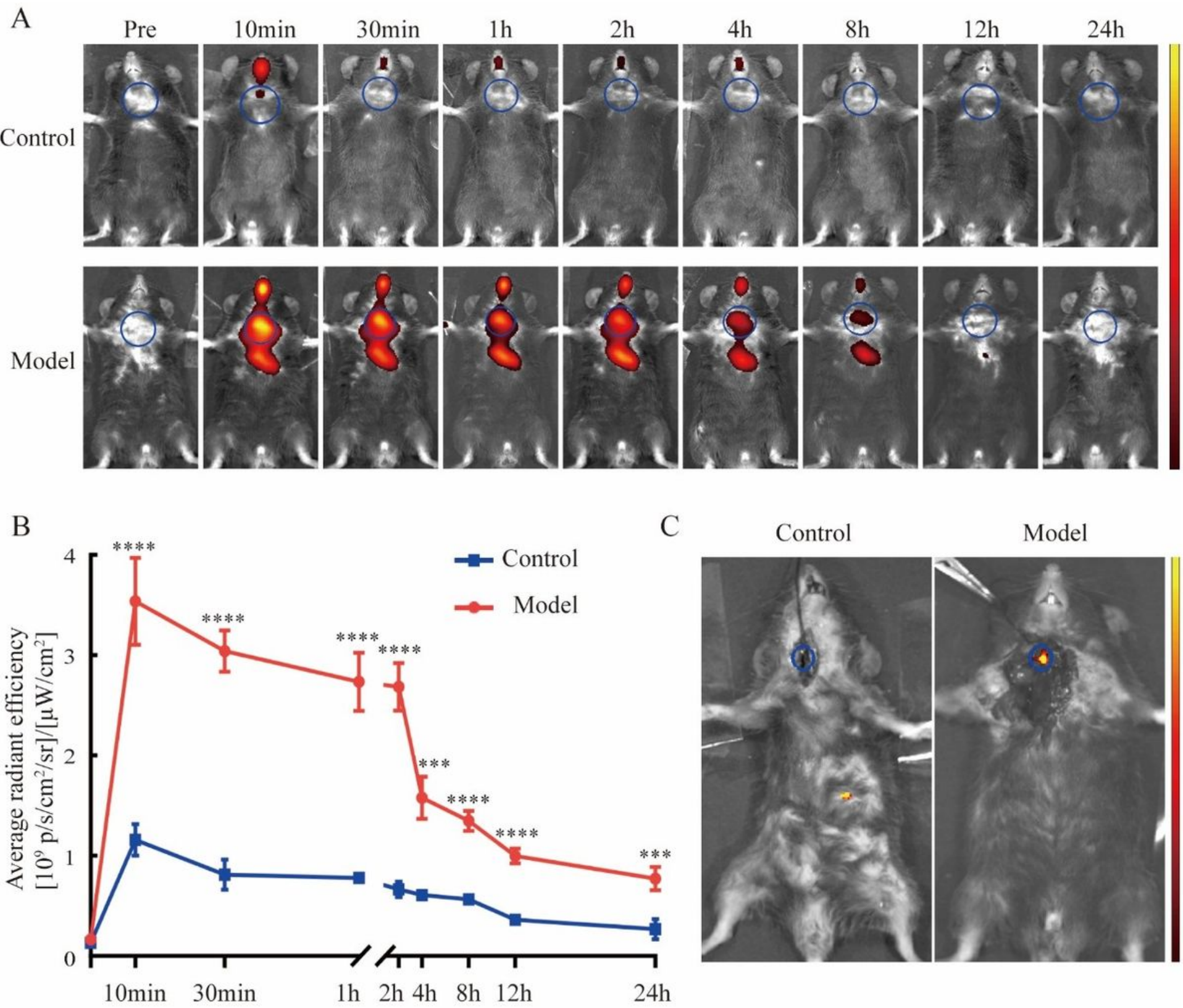

\section{Figure 5}

In vivo and ex vivo fluorescence imaging. (a) In vivo fluorescence imaging of carotid atherosclerosis model mice and control mice at the indicated time points after tail vein injection of AuNPs@PEG-PeptideCy5.5 (5 mg/kg). The colour bar indicates radiant efficiency (low, 0; high, $3.0 \times 109$ ). (b) Fluorescence intensity recorded at the carotid atherosclerotic site of the mice shown in (a). Data is presented as mean \pm S.D. for $n=3-6$ per group, ${ }^{\star \star \star} p<0.001,{ }^{\star \star \star *} p<0.0001$ by unpaired $t$-test. (c) Ex vivo NIR fluorescence images for surgical separation of the right carotid artery in atherosclerosis model and control mice after 
administration of AuNPs@PEG-Peptide-Cy5.5. The colour bar indicates radiant efficiency (low, 0; high, 3.0 $\times 109)$.

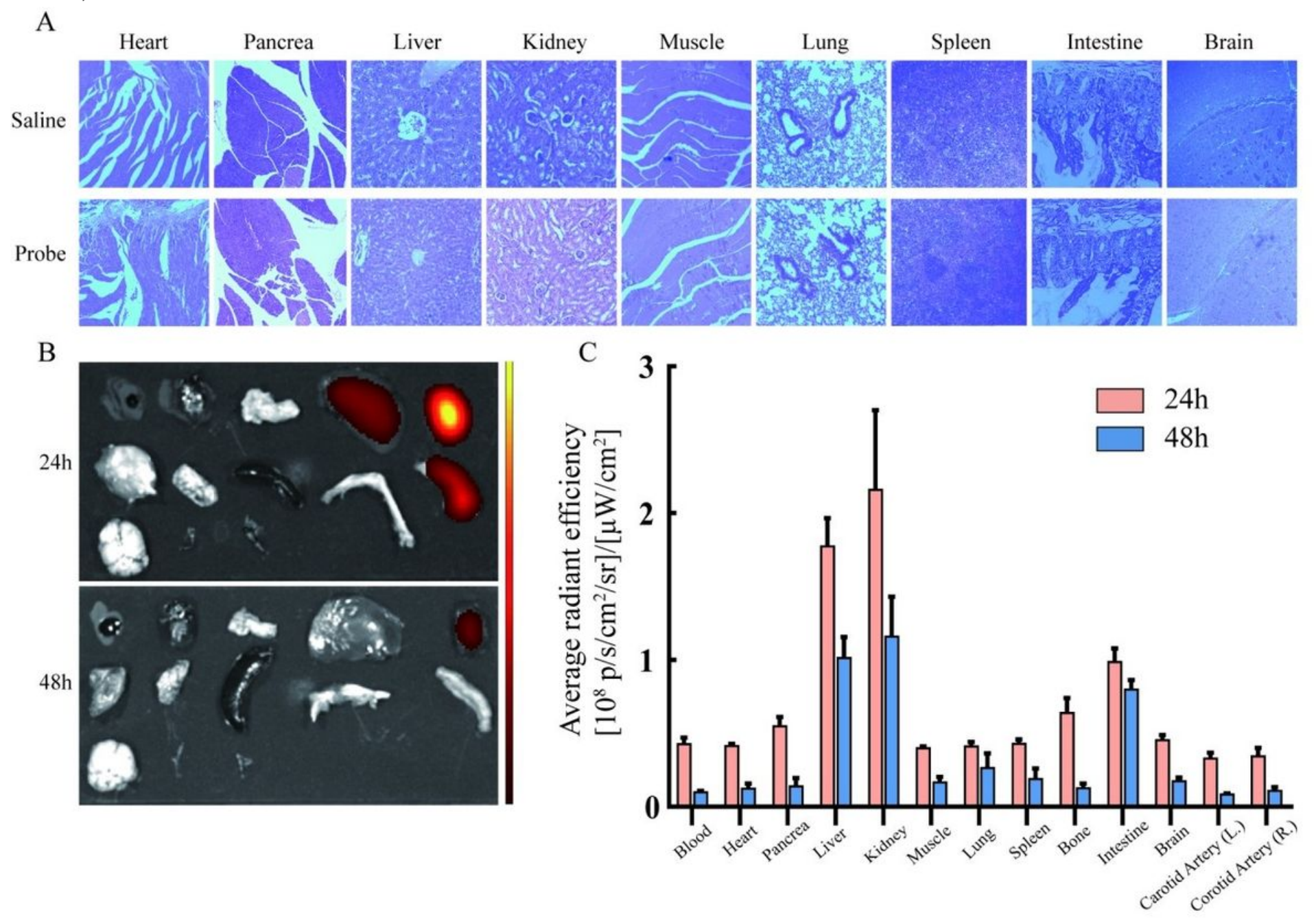

Figure 6

Organ toxicity and biodistribution post injection. (a) H\&E staining for pathological changes in heart, pancreas, liver, kidney, muscle, lung, spleen, intestine, and brain tissues at $24 \mathrm{~h}$ post-injection of model mice with saline and with AuNPs@PEG-Peptide-Cy5.5. (b) Representative images of dissected organs and tissues of model mice sacrificed at 24 and $48 \mathrm{~h}$ after intravenous injection of AuNPs@PEG-Peptide-Cy5.5. The colour bar indicates radiant efficiency (low, 0; high, $2.78 \times$ 108). (c) Biodistribution of AuNPs@PEGPeptide-Cy5.5 at 24 and $48 \mathrm{~h}$ post-injection. Data is presented as mean \pm S.D.

\section{Supplementary Files}

This is a list of supplementary files associated with this preprint. Click to download.

- Supplementaryfile.docx 